\title{
THE MAPPING CLASS GROUP OF A DISK WITH INFINITELY MANY HOLES
}

\author{
PAUL FABEL
}

\begin{abstract}
A left orderable completely metrizable topological group is exhibited containing Artin's braid group on infinitely many strands. The group is the mapping class group (rel boundary) of the closed unit disk with a sequence of interior punctures converging to the boundary. This resolves an issue suggested by work of Dehornoy.
\end{abstract}

\section{INTRODUCTION}

Artin's braid group $B_{n}$ is the fundamental group of the configuration space consisting of planar sets with precisely $n$ elements.

Artin's braid group on infinitely many strands $B_{\infty}$ is the direct limit of $B_{n}$ under monomorphisms $k_{n}: B_{n} \hookrightarrow B_{n+1}$ attaching a 'trivial strand'. Each element of $B_{\infty}$ can be seen as a countable collection of disjoint arcs in $[0,1] \times R^{2}$ connecting $\{0\} \times\{(1,0),(2,0), \ldots\}$ to $\{1\} \times\{(1,0),(2,0), .$.$\} such that all but finitely many of$ the arcs are line segments.

The group $B_{\infty}$ admits two natural incomplete topologies yielding distinct topological groups each of which is homeomorphic to the rational numbers.

In 4] a completely metrizable group $\overline{B_{\infty}}$ is constructed completing $B_{\infty}$ with the following topology: A sequence of braids $b_{n} \in B_{\infty}$ converges to $i d \in B_{\infty}$ iff for each $M$ there exists $N$ such that if $n \geq N$ then the first $M$ strands of $b_{n}$ determine the trivial braid on $M$ strands. A typical element of the completion is a 'wild braid' and is not determined by an ambient isotopy of the plane. The pure subgroup is precisely the inverse limit of Artin's pure braid groups.

However there is another natural topology on $B_{\infty}$ determined by a left ordering explored extensively in [2] [6], in which $b_{1}, b_{2}, \ldots$ converges to $i d$ iff for each $M$ there exists $N$ such that for $n \geq N$ the first $M$ strands of $b_{n}$ determine the trivial braid on $M$ strands and are unlinked from the remaining strands of $b_{n}$.

In reference to $B_{\infty}$ with this order topology, Dehornoy [2] has commented "... a group structure on the completion,.... would be more satisfying than the monoid structure of $E B_{\infty}$."

To this end, we exhibit a left ordered completely metrizable mapping class group $M\left(H_{\infty}\right)$ in which $B_{\infty}$ with the order topology from 2 is a densely embedded subgroup. Choosing a sequence $z_{n} \in \operatorname{int}\left(D^{2}\right)$ converging to a boundary point of a closed disk $D^{2} \subset R^{2}, H_{\infty}$ is the group of homeomorphisms of $D^{2}$ which fix $\partial D^{2}$ pointwise and which leave the set $\left\{z_{1}, z_{2}, \ldots\right\}$ invariant.

In contrast to $\bar{B}_{\infty}$ each element of $M\left(H_{\infty}\right)$ is a 'tame braid' determined by an isotopy of the disk. The natural map $j: M\left(H_{\infty}\right) \hookrightarrow \overline{B_{\infty}}$ is a continuous

Date: March 4, 2003.

1991 Mathematics Subject Classification. Primary 20F36, 57M60. 
monomorphism but not an embedding. Both $M\left(H_{\infty}\right)$ and $\bar{B}_{\infty}$ are homeomorphic to the irrational numbers.

Complications arise from the fact that $H_{\infty}$ is not locally connected. In particular the path component of the identity $H_{\infty}^{0}$ is not an open subgroup of $H_{\infty}$ and some care is required to verify (Lemma 2) that $H_{\infty}^{0}$ is in fact closed in $H_{\infty}$.

A general procedure is developed (Corollary 4) for generating a metrically complete group given a normal subgroup $H$ of a subgroup $G$ of the autohomeomorphism group of a metric compactum.

This determines the following complete metric $D$ on $M\left(H_{\infty}\right)$ :

Define $D\left(g H_{\infty}^{0}, f H_{\infty}^{0}\right)=D_{H}\left(g H_{\infty}^{0}, f H_{\infty}^{0}\right)+D_{H}\left(g^{-1} H_{\infty}^{0}, f^{-1} H_{\infty}^{0}\right)$ where $D_{H}$ is the (incomplete) Hausdorf metric determined by the condition

$D_{H}\left(p H_{\infty}^{0}, q H_{\infty}^{0}\right)<\varepsilon$ iff there exists $\hat{p}^{\wedge} \in p H_{\infty}^{0}$ and $q^{\wedge} \in q H_{\infty}^{0}$ such that $\left|p^{\wedge}(z)-q^{\wedge}(z)\right|<\varepsilon \forall z \in D^{2}$.

Dehornoy's left ordering on $B_{n}$ induces a left ordering on $M\left(H_{\infty}\right)$. It is verified in Theorem 2 that the order topology is compatible with the quotient topology of $M\left(H_{\infty}\right)$.

\section{BASIC DEFINITIONS AND NOTATION}

Throughout this paper all function spaces will have the compact open topology

and all identification spaces will have the quotient topology. $C(A, B)$ denotes the maps from $A$ to $B$, and $H(A, A)$ denotes the homeomorphisms from $A$ onto $A$.

If $G$ is a topological group then $G^{0} \subset G$ denotes the path component of the identity in $G$, and $M G$ denotes, mapping class group of $G$, the quotient space $G / G^{0}$.

Let $D^{2} \subset R^{2}$ denote the closed disk of radius 1 centered at $(1,0)$.

Let $Z_{n}=\left\{(1,0),\left(\frac{1}{2}, 0\right), . .\left(\frac{1}{n}, 0\right)\right\} \subset \operatorname{int}\left(D^{2}\right)$.

Let $Z=\cup_{n=1}^{\infty} Z_{n}$

Let $\alpha_{n}=\left\{(x, y) \in D^{2} \mid x=\frac{\frac{1}{n+1}+\frac{1}{n}}{2}\right\}$. Thus $\alpha_{n}$ is a vertical line segment halfway between $z_{n+1}$ and $z_{n}$.

Let $D_{n}=\left\{(x, y) \in D^{2} \mid \frac{\frac{1}{n+1}+\frac{1}{n}}{2} \leq x\right\}$.

Given $n>k$ let $D_{n, k}=\overline{D_{n} \backslash D_{k}}$.

Let $H_{\infty}=\left\{h \in H\left(D^{2}, D^{2}\right) \mid h(Z)=Z\right.$ and $\left.h_{\partial D^{2}}=i d_{\partial D^{2}}\right\}$.

Let $H_{n}=\left\{h \in H\left(D^{2}, D^{2}\right) \mid h\left(Z_{n}\right)=Z_{n}\right.$ and $\left.h_{\partial D^{2}}=i d_{\partial D^{2}}\right\}$.

Let $\hat{H_{n}}=\left\{h \in H_{n} \mid h_{D^{2} \backslash D_{n}}=i d_{D^{2} \backslash D_{n}}\right\}$

\section{ARTIN's BRAID GROUPS}

A braid $b_{n} \in B_{n}$ can be seen as the restriction to $n$ vertical line segments of a level preserving homeomorphism of the 3 -cell $D^{2} \times[0,1]$. Formally we select from several equivalent definitions (see [7 for a quick tour) and define $B_{n}$, Artin's braid group on $n$ strands to be the mapping class group $M\left(H_{n}\right)$. The following propositions are well known. See [1] for more details.

Proposition 1. The inclusion map $i_{n}: H_{n}^{\wedge} \rightarrow H_{n}$ induces an isomorphism $i_{n}^{*}$ : $M\left(H_{n}\right) \rightarrow M\left(H_{n}\right)$.

Proposition 2. The inclusion map $j_{n}: \hat{H_{n}} \rightarrow \hat{H_{n+1}}$ induces a monomorphism $j_{n}^{*}: M\left(\hat{H_{n}^{*}}\right) \hookrightarrow M\left(\hat{H_{n+1}}\right)$ 
Now define Artin's braid group on infinitely many strands $B_{\infty}$ as the direct limit of $B_{n}$ taken over the monomorphisms $k_{n}=i_{n+1}^{*} j_{n}^{*} i_{n}^{*-1}: B_{n} \hookrightarrow B_{n+1}$

Lemma 1. The monomorphisms $i_{n+1}^{*} j_{n}^{*} i_{n}^{*-1}: B_{n} \rightarrow B_{n+1}$ induce a monomorphism $i_{\infty}: B_{\infty} \hookrightarrow M\left(H_{\infty}\right)$.

Proof. By proposition 2 each element of $\lim _{\rightarrow} M\left(H_{n}\right)$ can be represented in the form $g H_{n}^{0}, g H_{n+1}^{0}, \ldots$ with $g \in H_{n}^{\wedge}$ and $g H_{n}^{0} \notin i m\left(k_{n-1}\right)$.

Thus $i_{\infty}\left(g H_{n}^{0}, g H_{n+1}^{0},\right)=g H_{\infty}^{0}$. Suppose $i_{\infty}\left(g H_{n}^{0}, g H_{n+1}^{0},\right)=g H_{\infty}^{0}=H_{\infty}^{0}$. Then $g \in H_{\infty}^{0} \subset \cap_{m=1}^{\infty} H_{m}^{0}$.

Thus $n=1$ and $\left(g H_{n}^{0}, g H_{n+1}^{0}, \ldots\right)=\left(H_{1}^{0}, H_{2}^{0}, \ldots\right)$. Hence $i_{\infty}$ is one to one.

\section{Building isotopies in $H_{\infty}$}

This section produces Corollaries 1 and 2

Remark 1 (Alexander trick). Any two homeomorphisms of $D^{2}$ which agree on $\partial D^{2}$ and a point $p \in \operatorname{int}\left(D^{2}\right)$ are isotopic rel $\partial D^{2} \cup p$.

Remark 2. Suppose $\alpha \subset D^{2} \backslash Z_{n}$ is a closed arc. Suppose $g_{t}: \alpha \hookrightarrow D^{2} \backslash Z_{n}$ is an isotopy such that $g_{0}=i d_{\alpha}, \partial\left(i m\left(g_{t}\right)\right) \subset \partial D^{2}$ and $\operatorname{int}\left(i m\left(g_{t}\right)\right) \subset \operatorname{int}\left(D^{2} \backslash Z_{n}\right)$. Then there exists an isotopy $G_{t}=D^{2} \rightarrow D^{2}$ such that $G_{0}=i d_{D^{2}},\left(G_{t}\right)_{\alpha}=g_{t}$ and $G_{t} \in H_{n}^{0}$.

Lemma 2. Suppose $g \in H_{\infty}, g_{D_{i}}=i d_{D_{i}}$ and $\forall n i d_{\alpha_{i+1}}$ is isotopic ( rel $\partial \alpha_{i+1}$ ) in $D^{2} \backslash Z_{n}$ to $g_{\alpha_{i+1}}$. Then there exists $f \in g H_{\infty}^{0}$ such that $f_{D_{i+1}}=i d_{D_{i+1}}$.

Proof. Choose $n>i+1$ such that $g\left(\alpha_{i+1}\right) \cap \alpha_{n}=\emptyset$. Let $\beta_{t}$ be an isotopy rel $\partial \alpha_{i+1}$ in $D^{2} \backslash Z_{n}$ such that $\beta_{0}=i d_{\alpha_{i+1}}$ and $\beta_{1}=g_{\alpha_{i+1}}$. By Remark 2 let $h_{t}$ be an isotopy in $H_{n}^{0}$ extending $\beta_{t}$ such that $h=i d_{D^{2}}$. Let $\phi: D^{2} \rightarrow D_{n, i}$ be a homeomorphism fixing pointwise $\alpha_{i+1} \cup\left(Z_{n} \backslash Z_{i}\right)$. Let $g_{t}^{*}=i d_{\left(D^{2} \backslash D_{n}\right)} \cup \phi h_{t} \phi^{-1} \cup i d_{\left(D_{k}\right)}$. Note $g_{t} \in H_{\infty}^{0}$ and $g_{t}$ fixes $D_{i}$ pointwise. Now consider $p_{t}=g\left(g_{t}^{*}\right)^{-1}$. Note $p_{0}=g$ and $p_{1}$ fixes $\alpha_{i+1} \cup \alpha_{i}$ pointwise. Hence by the Alexander trick we may isotop $p_{1}$ within $H_{\infty}^{0}$ to a map $f$ such that $f_{D_{i+1}}=i d_{D_{i+1}}$.

Corollary 1. Suppose $g \in H_{\infty}$ and for each $i \leq k$ and for each $n \alpha_{i} \in\left(g H_{n}^{0}\right)\left(\alpha_{i}\right)$. Then there exists $\hat{g} \in g H_{\infty}^{0}$ such that $\hat{g_{D_{k}}}=i d_{D_{k}}$.

Proof. Successively apply Lemma $2 k$ times to construct a map $\psi:[0, k] \rightarrow g H_{\infty}^{0}$ satisfying $\psi(t)_{D_{i}}=i d_{D_{i}}$ whenever $t \leq i$ and $i \in\{1,2, \ldots k\}$. Let $g \hat{=}=\psi(k)$.

Lemma 3. Suppose $h \in \cap H_{n}^{0}$ and $h_{D_{k}}=i d_{D_{k}}$. Then there exists an isotopy $h_{t}$ in $\cap H_{n}^{0}$ such that $h_{0}=h, h_{t\left(D_{k}\right)}=i d_{\left(D_{k}\right)}$ and $h_{1\left(D_{k+1}\right)}=i d_{\left(D_{k+1}\right)}$.

Proof. Choose $n>k+1$ such that $\alpha_{n} \cap h\left(\alpha_{k+1}\right)=\emptyset$. Let $g_{t}$ be a isotopy in $H_{n}^{0}$ such that $i d=g_{0}$ and $g_{1}=h$. Let $\phi: D^{2} \rightarrow \overline{D_{n} \backslash D_{k}}$ be a homeomorphism such that $\phi_{\alpha_{k+1} \cup\left(Z_{n} \backslash Z_{k}\right)}=i d_{\alpha_{k+1} \cup\left(Z_{n} \backslash Z_{k}\right)}$. Let $h_{t}^{*}=i d_{\left(D^{2} \backslash D_{n}\right)} \cup \phi g_{t} \phi^{-1} \cup i d_{D_{k}}$. Let $p_{t}=h\left(h_{t}^{*}\right)^{-1}$. Note $p_{1}$ fixes $\alpha_{k} \cup \alpha_{k+1}$ pointwise. Now isotop $p_{1\left(D_{k+1}, k\right)}$ to $i d_{D_{k+1}, k}$ via the Alexander trick while leaving $D_{k} \cup\left(D^{2} \backslash D_{k+1}\right)$ alone.

Corollary 2. $H_{\infty}^{0}$ is closed in $H_{\infty}$ and $\cap_{n=1}^{\infty} H_{n}^{0}=H_{\infty}^{0}$.

Suppose $h \in \cap H_{n}^{0}$. By repeated application of Lemma 3 construct a map $\psi$ : $[0, \infty) \rightarrow \cap H_{n}^{0}$ satisfying $\psi(0)=h$ and $\psi(t)_{D_{n}}=i d_{D_{n}}$ for $t \leq n$. Thus $\psi$ can be continuously extended to $[0, \infty]$ with $\psi(\infty)=i d$. Hence $\cap H_{n}^{0} \subset H_{\infty}^{0}$. Conversely 
$H_{\infty} \subset H_{n} \forall n$ and hence $H_{\infty}^{0} \subset H_{n}^{0} \forall n$. Hence $H_{\infty}^{0}=\cap_{n=1}^{\infty} H_{n}^{0}$. Moreover $H_{\infty}^{0}$ is closed in $H_{\infty}$ since its the intersection of closed sets $H_{n}^{0} \subset H\left(D^{2}, D^{2}\right)$.

\section{Recognizing COMPLETE QUOTIEnt GRoups.}

Expanding an elementary argument ( Proposition 1.3.10 [8]) that the autohomeomorphism $H(X, X)$ of a compact metric $X$ is completely metrizable, given a subgroup $G \subset H(X, X)$ with normal subgroup $H \triangleleft G$, we construct a completely metrizable group $(\bar{G} / \bar{H})$ such that if $G$ and $H$ are closed in $H(X, X)$ then $(\bar{G} / \bar{H})^{\wedge} \simeq G / H$. The proof of Theorem 1 makes use of the Baire category theorem, in a complete metric space the nested intersection of countably many open dense sets is dense, and in particular nonempty.

Remark 3. Suppose $(X, d)$ is a compact metric space. Fixing $\varepsilon>0$, a map $f \in C(X, X)$ is an $\varepsilon$ map if $\operatorname{diam}\left(f^{-1}(y)\right)<\varepsilon \forall y \in X$. The $\varepsilon$ maps form an open subspace of $C(X, X)$.

Lemma 4. Suppose $X$ is a compact metric space and $\left\{f, f_{1} f_{2}, . ., p_{1}, p_{2}, \ldots ..\right\} \subset$ $C(X, X)$. Suppose $f p_{n} \rightarrow i d$ and $p_{n} f_{n} \rightarrow i d$. Then $f_{n} \rightarrow f$.

Proof. Given $g \in C(X, X)$ denote the graph of $g$ as $\operatorname{gr}(g)=\{(x, y) \in X \times X \mid y=$ $g(x)\}$. Let $f_{n}^{*}$ be a subsequence of $f_{n}$ such that $\operatorname{gr}\left(f_{n}^{*}\right)$ converges (with respect to the Hausdorf metric over compacta in $X \times X$ ) to a compactum $\mathcal{X} \subset X \times X$. Let $p_{n}^{*}$ denote the corresponding subsequence of $p_{n}$. ( Thus if $f_{n}^{*}=f_{n_{m}}$ then $\left.p_{n}^{*}=p_{n_{m}}\right)$. Suppose $b=f(a)$. Let $a=p_{n}^{*} f_{n}^{*}\left(x_{n}\right)$. Then $x_{n} \rightarrow a$. Moreover $f(a)=$ $\lim f\left(p_{n}^{*} f_{n}^{*}\left(x_{n}\right)\right)=\lim \left(f p_{n}^{*}\right)\left(f_{n}^{*}\left(x_{n}\right)\right)=\lim f_{n}^{*}\left(x_{n}\right)$. This shows $g r(f) \subset \mathcal{X}$. Suppose $(a, b) \in \mathcal{X}$. Choose a subsequence $\hat{f_{n}}$ of $f_{n}^{*}$ such that $b=\lim \hat{f_{n}}(a)$. Let $\hat{p_{n}}$ denote the corresponding subsequence of $p_{n}$. Then $b=\lim \hat{f_{n}^{\wedge}}(a)=\lim \left(f p_{n}\right)\left(\hat{f_{n}}(a)\right)=$ $\lim f\left(\left(\hat{p_{n}} \hat{f_{n}}(a)\right)=\lim f(a)=f(a)\right.$. This shows every subsequential limit $\mathcal{X}$ of $g r\left(f_{n}\right)$ satisfies $\mathcal{X}=\operatorname{gr}(f)$. Hence $f_{n} \rightarrow f$.

Lemma 5. Suppose $G$ is a topological group with compatible metric d. Then the function $D: G \times G \rightarrow G$ defined via $D(f, g)=d(f, g)+d\left(f^{-1}, g^{1}\right)$ is a compatible metric on $G$.

Proof. Note $D(f, g)=0$ iff $d(f, g)+d\left(f^{-1}, g^{-1}\right)=0$ iff $f=g$ (since $d \geq 0$ ). Symmetry is immediate. $D(f, g)+D(g, p)=d(f, g)+d(g, p)+d\left(f^{-1}, g^{-1}\right)+$ $d\left(g^{-1}, p^{-1}\right) \geq d(f, p)+d\left(f^{-1}, p^{-1}\right)=D(f, p)$. To prove $D$ determines the same topology we will show that $(G, d)$ and $(G, d)$ have the same convergent sequences. If $D\left(g_{n}, g\right) \rightarrow 0$ then immediately $d\left(g_{n}, g\right) \rightarrow 0$ since $d \leq D$. Suppose $d\left(g_{n}, g\right) \rightarrow 0$. Then $d\left(g_{n}^{-1}, g^{-1}\right) \rightarrow 0$ since inversion is continuous in $G$. Thus $D\left(g, g_{n}\right) \rightarrow 0$.

Lemma 6. Suppose $h: X \rightarrow X$ is a homeomorphism of the compact metric space $(X, d)$. Then the map $\phi_{h}: C(X, X) \rightarrow C(X, X)$ defined via $\phi_{h}(f)=f h$ is an isometry with the uniform metric $D$ on $C(X, X)$.

Proof. Suppose $f \in C(X, X)$. Then $\phi_{h}\left(f h^{-1}\right)=f$. Thus $\phi_{h}$ is surjective. Suppose $f, g \in C(X, X)$ and $D(f, g)=d(f(x), g(x))$. Let $y=h^{-1}(x)$. Then $D(f h, g h) \geq$ $d(f h(y), d(g h(y)))=D(f, g)$.

Conversely suppose $D(f h, g h)=d(f h(y), g h(y))$. Then, letting $x=h(y), D(f, g) \geq$ $d(f(x), g(x))=D(f h, g h)$. 
Lemma 7. Suppose $H$ a group of isometries of the metric space $(Y, d)$. Then sets of the form $\overline{H(y)}$ determine a partition of $Y$. The function $\Pi: Y \rightarrow 2^{Y}$ defined via $\Pi(y)=\overline{H(y)}$ is a quotient map with respect to the Hausdorf metric D. Moreover $D(\overline{H(y)}, \overline{H(x)})<\varepsilon$ iff there exists $\hat{x} \in \overline{H(x)}$, and $\hat{y} \in \overline{H(y)}$ such that $d(\hat{x}, \hat{y})<$ $\varepsilon$.

Proof. Suppose $\hat{x} \in \overline{H(x)} \hat{y} \in \overline{H(y)}$ and $d(\hat{x}, \hat{y})<\varepsilon$. Choose $\hat{\varepsilon}$ such that $d(\hat{x}, \hat{y})<\varepsilon<\varepsilon$. Suppose $z \in \overline{H(x)}$. Suppose $\delta>0$. Choose $\{g, h, p\} \subset H$ such that $d(g(x), h(y))<\varepsilon$ and $d(z, p(x))<\delta$. Then $d\left(z, p g^{-1} h(y)\right) \leq d(z, p(x))+$ $d\left(p(x), p g^{-1} h(y)\right)<\delta+d\left(x, g^{-1} h(y)\right)=\delta+d(g(x), h(y))<\delta+\varepsilon$. Since $\delta$ was arbitrary this shows each point of $\overline{H(x)}$ is no further than $\varepsilon^{\wedge}$ from some point of $\overline{H(y)}$. By a symmetric argument we conclude $D(\overline{H(y)}, \overline{H(x)}) \leq \varepsilon<\varepsilon$. Thus $D(\overline{H(y)}, \overline{H(x)})<\varepsilon$ iff there exists $x^{\wedge} \in \overline{H(x)}$, and $y^{\wedge} \in \overline{H(y)}$ such that $d(\hat{x}, y)<$ $\varepsilon$. In particular if $z \in \overline{H(y)} \cap \overline{H(x)}$ then $D(\overline{H(y)}, \overline{H(x)})=0$ and hence $\overline{H(x)}=\overline{H(y)}$. Thus sets of the form $\overline{H(x)}$ determine a partition of $X$.

Finally, to prove $\Pi$ is a quotient map first observe that $\Pi$ is continuous since $D(\Pi(x), \Pi(y)) \leq d(x, y)$. Next suppose $\Pi^{-1}(A)$ is closed.

Suppose $D\left(\overline{\overline{H(x)}}, \overline{H\left(x_{n}\right)}\right) \rightarrow 0$ with $\overline{H\left(x_{n}\right)} \in A$. Choose $y_{n} \in \overline{H\left(x_{n}\right)}$ with $d\left(x, y_{n}\right) \rightarrow 0$. Thus $x \in \Pi^{-1}(A)$. Hence $\Pi(x)=\overline{H(x)} \in A$. Thus $A$ is closed.

Combining the previous two Lemmas shows that any group of homeomorphisms $H$ acting on a compact metric space $X$ generates a reasonable quotient of $C(X, X)$ denoted $C_{H}(X, X)$ as follows.

Corollary 3. Suppose $H$ is a group of homeomorphisms of the compact metric space $(X, d)$. Then, for each $f \in C(X, X)$, the sets of the form $\overline{f(H)}$ determine a partition of $C(X, X)$. If $C_{H}(X, X)$ denotes the associated quotient space then the Hausdorf metric is compatible with the quotient topology.

Proof. By Lemma $6 H$ acts isometrically on $C(X, X)$. Now apply Lemma 7

Throughout the remainder of this section $G$ is a group of homeomorphisms of the compact metric space $X, H$ is a normal subgroup of $G$ and $\bar{G}$ denotes the closure of $G$ in $C(X, X) \cdot \bar{G} / \bar{H}$ denotes the subspace of $C_{H}(X, X)$ consisting of elements of the form $\overline{f H}$ with $f \in \bar{G}$.

Lemma 8. Define $*: \bar{G} / \bar{H} \times \bar{G} / \bar{H} \rightarrow \bar{G} / \bar{H}$ via $*((\overline{f H}),(\overline{p H}))=\overline{f p H}$. Then $*$ is well defined, continuous, and determines that $\bar{G} / \bar{H}$ is a monoid.

Proof. To prove $*$ is well defined suppose $\hat{f} \in(\overline{f H})$ and $\hat{p} \in(\overline{p H})$. It suffices to show $f^{\wedge} p \in \overline{f p H}$. Choose $\left\{f_{n}, p_{n}\right\} \in G$ with $f_{n} \rightarrow f$ and $p_{n} \rightarrow p$. Thus $\overline{f_{n} H} \rightarrow \overline{f H}$ and $\overline{p_{n} H} \rightarrow \overline{p H}$. Since $f_{n} H$ is dense in $\overline{f_{n} H}$ we may choose $f_{n}^{\wedge} \in f_{n} H$ and $\hat{p_{n}} \in p_{n} H$ with $\hat{f_{n}} \rightarrow \hat{f}$ and $\hat{p_{n}} \rightarrow \hat{p}$. Let $\hat{f_{n}}=f_{n} h_{n}$ and $\hat{p_{n}}=p_{n} h^{n}$ with $\left\{h_{n}, h^{n}\right\} \in H$. Then,since $H$ is normal $\hat{f_{n}} \hat{p_{n}}=\left(f_{n} h_{n}\right) p_{n} h^{n}=f_{n} p_{n}\left(p_{n}^{-1} h_{n} p_{n}\right) h^{n} \in f_{n} p_{n} H$. Note $\overline{\left(f_{n} p_{n}\right) H} \rightarrow \overline{f p H}$ since $f_{n} p_{n} \rightarrow f p$. Thus $\hat{f} \hat{p}=\lim \hat{f_{n}^{\wedge}} \hat{p_{n}} \in \overline{f p H}$. Hence $*$ is well defined. To prove $*$ is continuous suppose $\overline{f_{n} H} \rightarrow \overline{f H}$ and $\overline{p_{n} H} \rightarrow \overline{p H}$. Choose $\hat{f_{n}} \in \overline{f_{n} H}$ and $\hat{p_{n}} \in \overline{p_{n} H}$ such that $\hat{f_{n}} \rightarrow f$ and $\hat{p_{n}} \rightarrow p$. Thus $\hat{\hat{f_{n} \hat{p_{n} H}}} \rightarrow \overline{f p H}$. Note $\bar{H} * \overline{f H}=\overline{H f H}=\overline{f H} * \bar{H}$.

Corollary 4. Let $(\bar{G} / \bar{H})^{\wedge}$ denote the subspace of $\bar{G} / \bar{H}$ consisting of invertible elements. (i.e. $\overline{f H} \in(\bar{G} / \bar{H})^{\wedge}$ iff there exists $\overline{p H} \in \bar{G} / \bar{H}$ such that $\overline{p f H}=\overline{f p H}=\bar{H}$ ) . Then $(\bar{G} / \bar{H})$ is a completely metrizable topological group. 
Proof. $(\bar{G} / \bar{H})^{\wedge}$ is a group since $\bar{G} / \bar{H}$ is a monoid. Note elements of the form $\overline{g H}$ with $g \in G$ are dense in $(\bar{G} / \bar{H})^{\wedge}$. Thus to prove inversion in $(\bar{G} / \bar{H})^{\wedge}$ is continuous we need only check the case $f_{n} \rightarrow f, f_{n} \in G, \overline{f_{n} H} \rightarrow \overline{f H}$, and $\overline{f p H}=\overline{f p H}=\bar{H}$.

We must show $\overline{f_{n}^{-1} H} \rightarrow \overline{p H}$. Note $d\left(f, f_{n}\right)=d\left(f f_{n}^{-1}, i d\right)$. Thus $\overline{f f_{n}^{-1} H} \rightarrow \bar{H}$. Hence $\overline{f_{n}^{-1} H}=\overline{p f H} * \overline{f_{n}^{-1} H}=\overline{p f f_{n}^{-1} H} \rightarrow \overline{p H}$. Let $D$ denote the Hausdorf metric on $(\bar{G} / \bar{H})^{\wedge}$. Define as in Lemma [5 a metric $D^{\wedge}$ on $(\bar{G} / \bar{H})^{\wedge}$ via $D^{\wedge}(\overline{f H}, \overline{g H})=$ $D(\overline{f H}, \overline{g H})+D\left((\overline{f H})^{-1},(\overline{g H})^{-1}\right)$.

Suppose $\overline{f_{n} H}$ is $D^{\wedge}$ Cauchy. Let $\overline{f_{n} H} \rightarrow \overline{f H}$ and $\left.\overline{\left(f_{n} H\right.}\right)^{-1} \rightarrow \overline{g H}$ with $\left.\overline{\{f H}, \overline{g H}\right\} \subset$ $C_{H}(X, X)$. Then $\overline{f_{n} H} *\left(\overline{f_{n} H}\right)^{-1} \rightarrow \overline{f H} * \overline{g H}=\bar{H}$. Similarly $\overline{g H} * \overline{f H}=\bar{H}$. Hence $\overline{\{f H}, \overline{g H}\} \subset(\bar{G} / \bar{H})^{\wedge}$.

Theorem 1. The function $\phi: G / H \rightarrow(\bar{G} / \bar{H})^{\wedge}$ defined via $\phi(g H)=\overline{g H}$ is a homomorphism. If $G$ is closed in $H(X, X)$ then $\phi$ is an epimorphism. If $H$ is closed in $G$ then $\phi$ is a monomorphism and an embedding. Consequently if $H$ is closed in $G$ and $G$ is closed in $H(X, X)$ then $\phi$ is a homeomorphism and in particular $G / H$ is completely metrizable.

Proof. $\phi$ is well defined since by Corollary 3 sets of the form $\overline{f H}$ determine a partition of $C(X, X)$. To show $\phi$ is a homomorphism first note $\phi\left(g_{1} H\right)\left(g_{2} H\right)=$ $\phi\left(g_{1} g_{2} H\right)=\overline{g_{1} g_{2} H}$. However by Lemma $8 \overline{g_{1} g_{2} H}=*\left(\overline{g_{1} H}, \overline{g_{2} H}\right)$.

Suppose $G$ is closed in $H(X, X)$ and $\overline{f H} \in(\bar{G} / \bar{H})^{\wedge}$. Let $\overline{p H} \in(\bar{G} / \bar{H})^{\wedge}$ satisfy $\overline{p f H}=\overline{f p H}=\bar{H}$. Choose a sequence $p_{n} \in \overline{p H}$ such that $f p_{n} \rightarrow i d$. Choose a sequence $f_{n} \in \overline{f H}$. Such that $p f_{n} \rightarrow i d$. By Lemma $4 f_{n} \rightarrow f$. Fixing $\varepsilon>0$ since $p_{n} f_{n} \rightarrow i d$ it follows that $f_{n}$ is eventually an $\varepsilon$ map.

Thus, those maps of $\frac{f H}{f H}$ which are $\varepsilon$ maps form an open and dense subspace of $\overline{f H}$. Since this holds for each $\varepsilon$, by the Baire category theorem there is a dense subset of homeomorphisms within $\overline{f H}$. In particular since $G=\bar{G}$ then $G \cap \overline{f H} \neq \emptyset$. Choosing $g \in G \cap \overline{f H}$ we have $\phi(g H)=\overline{g H}=\overline{f H}$.

Suppose $H$ is closed in $G$. Then the closure of $g H$ within $G$ is $g H$ and therefore we apply Lemma 7 to obtain a Hausdorf metric $d_{1}$ on $G / H$ inherited from the uniform metric of $G$. By Cor 3 we may use a Hausdorf metric $d_{2}$ on $(\bar{G} / \bar{H})^{\wedge}$ inherited from the uniform metric of $\bar{G}$. Note $d_{1}\left(g_{1} H, g_{2} H\right)=d_{2}\left(\overline{g_{1} H}, \overline{g_{2} H}\right)=d_{2}\left(\phi\left(g_{1} H\right), \phi\left(g_{2} H\right)\right)$ Thus if $H$ is closed in $G$ then $\phi$ is an embedding.

Corollary 5. $M\left(H_{\infty}\right)$ admits a compatible complete metric

$$
D: M\left(H_{\infty}\right) \times M\left(H_{\infty}\right) \rightarrow M\left(H_{\infty}\right)
$$

determined by the condition $D\left(f H_{\infty}^{0}, g H_{\infty}^{0}\right)<\varepsilon$ iff there exists $f_{1} \in f H_{\infty}^{0}, f_{2} \in$ $f^{-1} H_{\infty}^{0}, g_{1} \subset g H_{\infty}^{0}$ and $g_{2} \in g^{-1} H_{\infty}^{0}$ such that $d\left(f_{1}, g_{1}\right)<\varepsilon$ and $d\left(f_{2}, g_{2}\right)<\varepsilon$. (Note $d(p, q)<\varepsilon$ iff $|p(x)-q(x)|<\varepsilon \forall x \in D^{2}$ ).

Proof. Observe by definition $H_{\infty}$ is closed in $H\left(D^{2}, D^{2}\right)$. By Lemma $2 H_{\infty}^{0}=\cap H_{n}^{0}$ and hence $H_{\infty}^{0}$ is closed in $H_{\infty}$ since each of $H_{n}^{0}$ is closed in $H\left(D^{2}, D^{2}\right)$. Now apply Theorem 1 and harness the metric from the proof of Corollary 4

\section{A Left ORDERING ON $M\left(H_{\infty}\right)$ COMPATIBLE With its TOPOLOGY}

An ordered group is left ordered if $f<g \Rightarrow h f<h g$. In [5], 9] Dehornoy's left ordering [3] on $B_{n}$ is interpreted geometrically. Letting $\gamma_{i} \subset D^{2}$ denote the line segment connecting $z_{i}$ and $z_{i+1}$, the question of whether a given braid $g H_{n}^{0} \neq H_{n}^{0}$ is positive or negative is settled at the smallest index $i$ for which $\gamma_{i} \notin\left(g H_{n}^{0}\right)\left(\gamma_{i}\right)$. 
Consequently, (recalling the monomorphism $k_{n}: B_{n} \hookrightarrow B_{n+1}$ ) for each $n$ there exists a left ordering $<_{n}$ on $B_{n}$ satisfying the following conditions:

(1) If $f H_{n}^{0}<_{n} g H_{n}^{0}$ then $k_{n}\left(f H_{n}^{0}\right)<_{n+1} k_{n}\left(g H_{n}^{0}\right)$

(2) If $f H_{n}^{0}<_{n} g H_{n}^{0}$ and $p H_{n}^{0} \in B_{n}$ then $p f H_{n}^{0}<_{n} p g H_{n}^{0}$

(3) If $f H_{n}^{0} \neq g H_{n}^{0}$ then the question of whether $f H_{n}^{0}<_{n} g H_{n}^{0}$ or $g H_{n}^{0}<_{n} f H_{n}^{0}$ is settled by inspection of the arc isotopy classes $\left(f H_{n}^{0}\right)\left(\alpha_{i}\right)$ and $\left(g H_{n}^{0}\right)\left(\alpha_{i}\right)$ for the smallest index $i$ satisfying $\left(f H_{n}^{0}\right)\left(\alpha_{i}\right) \neq\left(g H_{n}^{0}\right)\left(\alpha_{i}\right)$.

Theorem 2. There exists a left ordering on $M\left(H_{\infty}\right)$ such that the order topology is compatible with the quotient topology.

Proof. Given $\left\{g H_{\infty}^{0}, f H_{\infty}^{0}\right\} \subset M\left(H_{\infty}\right)$ declare $g H_{\infty}^{0}<f H_{\infty}^{0}$ iff there exists $n$ such that $g H_{n}^{0}<_{n} f H_{n}^{0}$. Note by Lemma 2 given $\left\{f H_{\infty}^{0}, g H_{\infty}^{0}\right\} \subset M\left(H_{\infty}\right)$ then $f H_{\infty}^{0}=$ $g H_{\infty}^{0}$ iff $f H_{n}^{0}=g H_{n}^{0} \forall n$. It follows easily that $<$ is a left ordering on $M\left(H_{\infty}^{0}\right)$. For compatibility suppose $g \in\left(g_{1} H_{\infty}^{0}, g_{2} H_{\infty}^{0}\right)$, an open interval in the order topology. Choose $n$ such that $g_{1} H_{n}^{0}<g H_{n}^{0}<g_{2} H_{n}^{0}$ and $g_{2}^{-1} H_{n}^{0}<g^{-1} H_{n}^{0}<g_{1}^{-1} H_{n}^{0}$. Choose $\delta>0$ such that for $f \in H_{n}$ if $d(f, g)<\delta$ and $d\left(f^{-1}, g^{-1}\right)<\delta$ then $f H_{n}^{0}=g H_{n}^{0}$. Taking the metric $D$ on $M\left(H_{\infty}^{0}\right)$ from Corollary 5 it follows that the open metric ball $B\left(g H_{\infty}^{0}, \delta\right) \subset\left(g_{1} H_{\infty}^{0}, g_{2} H_{\infty}^{0}\right)$.

Conversely suppose $U$ is open in $M\left(H_{\infty}\right)$. Let $q H_{\infty}^{0} \in U$.

Let $V=\left\{q^{-1} h H_{\infty}^{0} \mid h H_{\infty}^{0} \in U\right\}$. Note $V$ is open ( since $M\left(H_{\infty}^{0}\right)$ is a topological group) and $H_{\infty}^{0} \in V$. Choose $\varepsilon>0$ such that $B_{D}\left(H_{\infty}^{0}, \varepsilon\right) \subset V$.

Choose $k$ such that $\operatorname{diam}\left(D^{2} \backslash D_{k}\right)<\varepsilon$. Choose $f H_{\infty}^{0} \in B_{D}\left(H_{\infty}^{0}, \varepsilon\right)$ such that $f_{D_{k}}=i d_{D_{k}}$.

Suppose $f^{-1} H_{\infty}^{0}<g H_{\infty}^{0}<f H_{\infty}^{0}$. Then for each $n f^{-1} H_{n}^{0} \leq g H_{n}^{0} \leq f H_{n}^{0}$.

Suppose $1 \leq i \leq k$ and $n$ is any positive integer. Then

$$
\left(f H_{n}^{0}\right)\left(\alpha_{i}\right)=\left(f^{-1} H_{n}^{0}\right)\left(\alpha_{i}\right)=H_{n}^{0}\left(\alpha_{i}\right) .
$$

Hence $H_{n}^{0}\left(\alpha_{i}\right)=g H_{n}^{0}\left(\alpha_{i}\right)$ since otherwise we contradict one of $f^{-1} H_{n}^{0} \leq g H_{n}^{0}$ or $g H_{n}^{0} \leq f H_{n}^{0}$.

Thus $\forall n$ and each $i \leq k \alpha_{i} \in\left(g H_{n}^{0}\right)\left(\alpha_{i}\right)$. By Corollary 1 select $g \wedge \in H_{\infty}^{0}$ such that $\hat{g_{D_{k}}}=i d_{D_{k}}$. Hence $D\left(g H_{\infty}^{0}, H_{\infty}^{0}\right)<\varepsilon$.

This shows the open interval $\left(f^{-1} H_{\infty}^{0}, f H_{\infty}^{0}\right) \subset V$. Hence (since left composition preserves the ordering) the open interval $\left(q f^{-1} H_{\infty}^{0}, q f H_{\infty}^{0}\right) \subset U$.

Thus the order topology of $M\left(H_{\infty}\right)$ is compatible with the quotient topology.

\section{REFERENCES}

[1] Birman, Joan S. Braids, links, and mapping class groups. Annals of Mathematics Studies, No. 82. Princeton University Press, Princeton, N.J.; University of Tokyo Press, Tokyo, 1974. $\mathrm{ix}+228 \mathrm{pp}$.

[2] Dehornoy, Patrick "Transfinite braids and left distributive operations". Math. Z. 228 (1998), no. 3, 405-433.

[3] Dehornoy, Patrick "Braid groups and left distributive operations." Trans. Amer. Math. Soc. 345 (1994), no. 1, 115-150.

[4] Fabel, Paul. "Completing Artin's braid group on infinitely many strands." Preprint. http://front.math.ucdavis.edu/math.GT/0201303

[5] Fenn, R.; Greene, M. T.; Rolfsen, D.; Rourke, C.; Wiest, B. "Ordering the braid groups." Pacific J. Math. 191 (1999), no. 1, 49-74.

[6] Funk, Jonathon "The Hurwitz action and braid group orderings." CT2000 Conference (Como). Theory Appl. Categ. 9 (2001/02), 121-150

[7] Rolfsen, Dale "New developments in the theory of Artin's braid groups" Topology and Applications (to appear) 
[8] van Mill, J. Infinite-dimensional topology. Prerequisites and introduction. North-Holland Mathematical Library, 43. North-Holland Publishing Co.Amsterdam, 1989

[9] Wiest, Bert "Dehornoy's ordering of the braid groups extends the subword ordering." Pacific J. Math. 191 (1999), no. 1, 183-188.

Drawer MA, Department of Mathematics and Statistics, Mississippi State, Ms. 39762

E-mail address: fabel@ra.msstate.edu

URL: http://www2.msstate.edu/ fabel/ 